\title{
Sentinel Surveillance: A Reliable Way To Track Antibiotic Resistance in Communities?
}

\author{
Stephanie J. Schrag, ${ }^{*}$ Elizabeth R. Zell, ${ }^{*}$ Anne Schuchat, ${ }^{*}$ and Cynthia G. Whitney*
}

\begin{abstract}
We used population-based data to evaluate how often groups of randomly selected clinical laboratories accurately estimated the prevalence of resistant pneumococci and captured trends in resistance over time. Surveillance for invasive pneumococcal disease was conducted in eight states from 1996 to 1998. Within each surveillance area, we evaluated the proportion of all groups of three, four, and five laboratories that estimated the prevalence of penicillin-nonsusceptible pneumococci (\%PNSP) and the change in \%PNSP over time. We assessed whether sentinel groups detected emerging fluoroquinolone resistance. Groups of five performed best. Sentinel groups accurately predicted \%PNSP in five states; states where they performed poorly had high between-laboratory variation in \%PNSP. Sentinel groups detected large changes in prevalence of nonsusceptibility over time but rarely detected emerging fluoroquinolone resistance. Characteristics of hospital-affiliated laboratories were not useful predictors of a laboratory's \%PNSP. Sentinel surveillance for resistant pneumococci can detect important trends over time but rarely detects newly emerging resistance profiles.
\end{abstract}

A ntibiotic-resistant infections are an emerging problem in community as well as nosocomial settings. Streptococcus pneumoniae infections are a leading cause of communityacquired respiratory illness in young children, the elderly, and persons with chronic medical conditions. Pneumococcal infections range from otitis media and bacteremia to pneumonia and meningitis. Although penicillin has traditionally been an effective treatment for pneumococcal infections, in recent years the increasing prevalence of drug-resistant pneumococci threatens the effectiveness of antibiotic therapy $(1,2)$.

Surveillance for resistant pneumococci is an essential component of public health efforts to prevent the spread of drug resistance. In addition to increasing awareness of the public and health-care providers about resistance, surveillance data can be used to target high-prevalence areas for judicious use of antibiotics, pneumococcal vaccination campaigns, or both; identify newly emerging strains and resistance profiles; and assess trends in resistance. At the national level, surveillance data can contribute to the development of clinical guidelines for managing pneumococcal disease $(3,4)$. Local surveillance data can in some instances guide patient care (4).

The prevalence of drug-resistant pneumococci varies geographically. Because national trends may not reflect trends within specific regions, local and state-specific data can motivate prevention efforts (5). Although invasive disease due to drug-resistant pneumococci was added to the National Notifiable Diseases List in 1994, mandatory reporting remains low (53\% of states and territories in 1999) (6), in part because collecting antimicrobial susceptibility data can be difficult. Active, population-based surveillance for resistant pneumo-

${ }^{*}$ Centers for Disease Control and Prevention, Atlanta, Georgia, USA cocci based on laboratory-confirmed invasive disease may be considered the most accurate method of estimating rates of drug-resistant pneumococcal disease in a defined area. Such systems, however, are often costly and labor-intensive for state or local health departments to maintain.

Sentinel surveillance, a system that collects information on drug-resistant pneumococci from a limited sample of hospital, clinic, and/or private laboratories, has been suggested as a feasible alternative method of collecting regional data, and some states are adopting this approach (7). Although sentinel systems are useful for monitoring trends in a number of diseases (8-10) and a sentinel hospital surveillance system in the 1980s first detected increases in the prevalence of penicillin-resistant pneumococci in the United States (11), observations that the prevalence of resistant pneumococcal isolates can vary dramatically from laboratory to laboratory within a state or area (12) raise the question of whether sentinel laboratories can accurately reflect an area's prevalence of pneumococcal resistance.

For pneumococcus, the most common approach to sentinel surveillance is to select a small number of clinical laboratories within an area and collect information on susceptibility of all invasive pneumococcal isolates at those facilities as a way of estimating the prevalence of resistance in the area as a whole. To evaluate the validity of this sentinel approach, we assessed how often small groups of laboratories in a given area accurately estimated the area's proportion of resistant invasive pneumococcal isolates, using population-based surveillance as the standard. We also evaluated whether such sentinel groups of laboratories accurately tracked changes in the proportion of drug-resistant pneumococci over time, and whether they could detect newly emerging resistance profiles. Finally, we 
explored whether hospital characteristics could be used to guide selection of hospital laboratories for inclusion in sentinel systems, in order to increase the system's representativeness and reliability.

\section{Methods}

\section{Population-Based Data}

Invasive pneumococcal surveillance was conducted from 1996 to 1998 as part of the Active Bacterial Core Surveillance/ Emerging Infections Program Network (ABCs) using previously described methods (1). Briefly, project personnel communicated at least twice each month with contacts in all participating microbiology laboratories serving acute-care hospitals in San Francisco County, California; Connecticut; eight counties in Georgia (Cobb, Clayton, De Kalb, Douglas, Fulton, Gwinnett, Newton, and Rockdale) with 12 additional Atlanta-area counties starting in 1997; six counties in Maryland (Anne Arundel, Baltimore, Baltimore City, Carroll, Harford, and Howard); seven counties in Minnesota (Anoka, Carver, Dakota, Hennepin, Ramsey, Scott, and Washington); seven counties in New York starting in 1997 (Genesee, Livingston, Monroe, Ontario, Orleans, Wayne, and Yates); three counties in Oregon (Clackamas, Multnomah, and Washington); and five counties in Tennessee (Davidson, Hamilton, Knox, Shelby, and Williamson).

A case was defined as the isolation of Streptococcus pneumoniae from a normally sterile site (e.g., blood or cerebrospinal fluid) from a resident of a surveillance area. Periodic audits were conducted in each area. Any cases newly identified by audits were included in the surveillance database.

All isolates were sent to one of two centralized laboratories for susceptibility testing by broth microdilution, with a panel of drugs that included (in 1998) penicillin, amoxicillin, cefotaxime, cefuroxime, meropenem, erythromycin, clindamycin, chloramphenicol, vancomycin, rifampin, levofloxacin, trovafloxacin, and quinupristin-dalfopristin (Synercid7). Nonsusceptibility (resistance and intermediate susceptibility) was determined according to criteria of the National Committee for Clinical Laboratory Standards (13).

\section{Ability of Sentinel Laboratory Groups To Estimate Proportion of Resistant Isolates}

In each surveillance area for 1998, we generated all possible simple random samples of three, four, and five laboratories, excluding laboratories with $<10$ isolates. We limited our selection to up to five laboratories because a central objective of sentinel surveillance is to reduce required resources by reducing the number of facilities participating in the surveillance system. We refer to these simple random samples as sentinel groups of laboratories. We then calculated the percent of penicillin-nonsusceptible (MIC $\geq 0.1 \mu \mathrm{g} / \mathrm{mL}$ ) pneumococci (\%PNSP) among isolates in each of these sentinel groups and compared these percentages to the area's actual \%PNSP, as measured by ABCs. The \%PNSP in sentinel groups was considered to be accurate if it was within 5 percentage points of the area's actual \%PNSP. We chose this interval because variation in the \%PNSP within this range is unlikely to influence public health decisions (12).

We used a finite population correction based on the total number of isolates in each surveillance area to assess the number of randomly sampled isolates that would be needed to estimate an area's actual \%PNSP within 5 percentage points (14). We compared that number with the number of isolates in sentinel groups in each area.

\section{Ability of Sentinel Groups To Track Changes in Prevalence of Drug-Resistant Pneumococci over Time}

In each surveillance area, we subtracted the \%PNSP in each possible group of five laboratories in 1996 from that measured for the group of five laboratories in 1998. We included only laboratories with $\geq 10$ isolates in each of the 2 -year periods. We then measured how often the change in \%PNSP in sentinel groups was within 5 percentage points of the area's actual change in \%PNSP during the same time periods, based on $\mathrm{ABCs}$ data. We performed a similar analysis using the percentage of erythromycin-nonsusceptible ( $\mathrm{MIC} \geq 0.5 \mu \mathrm{g} / \mathrm{mL}$ ) isolates as the outcome measure.

\section{Ability of Sentinel Goups To Detect Emerging Fluoroquinolone Resistance}

Using data from 1998, we measured the proportion of all possible groups of five sentinel laboratories within each surveillance area that captured any pneumococcal isolates with fluoroquinolone (levofloxacin or trovafloxacin) nonsusceptibility. We then compared that proportion with area-specific data on the presence of pneumococcal fluoroquinolone resistance from ABCs in 1998.

\section{Evaluation of Hospital Predictors of \%PNSP}

We merged ABCs data from 1997 and 1998 with purchased data on hospital characteristics collected by the American Hospital Association (AHA) as part of the AHA Annual Survey of Registered American Hospitals in 1997. We categorized each hospital that matched between the two datasets into the following PNSP classes: $\geq 5$ percentage points above the surveillance area proportion PNSP (high PNSP), $<5$ percentage points above or below the surveillance area PNSP (average PNSP), or $\geq 5$ percentage points below the surveillance area PNSP (low PNSP). We used logistic regression to perform univariate analyses. We compared hospital characteristics in the high group with those in the average group, separately comparing hospital characteristics in the low group with those in the average group. We categorized continuous variables according to their quartiles or medians based on their distributions. We limited our analysis to hospital characteristics that might plausibly influence a hospital's \%PNSP based on findings of previous studies $(15,16)$. 


\section{Results}

\section{Population-Based Data}

The \%PNSP across surveillance areas in 1998 varied from 15 (California and New York) to 35 (Tennessee) (Table 1). The number of laboratories that isolated invasive pneumococci and the total number of invasive pneumococcal isolates also varied by surveillance area (Table 1). Consistent with previous observations (12), each surveillance area had striking variation across laboratories in the \%PNSP in invasive pneumococcal isolates (Figure).

\section{Ability of Sentinel Laboratory Groups To Estimate \%PNSP}

In New York, California, and Oregon (areas with a relatively small number of laboratories with $\geq 10$ invasive pneumococcal isolates), sentinel groups of three, four, or five laboratories all did well at estimating the area's actual \%PNSP (Table 1). In the remaining areas, increasing the number of laboratories included in sentinel groups from three to five increased the probability that the sentinel \%PNSP approached the area's actual \%PNSP. However, in Georgia and Tennessee, the two areas with the highest actual \%PNSP, sentinel groups of five laboratories still poorly estimated the area's actual percentage (Table 1).

In surveillance areas where most sentinel groups had an adequate sample size to estimate \%PNSP accurately (i.e., the number of isolates met the sample size requirement), sentinel groups performed well compared with population-based surveillance (Table 2). In contrast, in Georgia and Tennessee, where sentinel groups performed poorly, a smaller proportion of sentinel groups met the minimum sample size requirements. However, in some states that failed to meet sample size requirements (e.g., Connecticut), sentinel groups performed well.

\section{Ability of Sentinel Groups To Detect Trends in Prevalence of Nonsusceptible Pneumococci}

The actual change in \%PNSP in 1998 compared with that in 1996 varied across areas, ranging from Georgia's 2\% decline to Maryland's 7\% increase (Table 3). Because sentinel groups of five were the most accurate at predicting an area's actual \%PNSP, we focused strictly on groups of five for this analysis. Laboratories participating in ABCs in 1998 were often not the same as those participating in 1996 because of hospital or laboratory mergers, closing or opening of microbiology facilities in the surveillance areas, and expansion of areas under surveillance. Consequently, only a subset of all possible sentinel groups in 1998 matched those in 1996.

Over two thirds of each area's sentinel groups of five accurately estimated changes in \%PNSP, except in Tennessee, where only $45 \%$ correctly estimated a $<5$ percentage point change (Table 3). In the three areas with large changes in \%PNSP $(\geq 3$ percentage points), $>90 \%$ of sentinel groups in each area predicted the direction of the change (increases in each case).

Trends in the proportion of isolates that were erythromycin nonsusceptible also varied by area, and three areas showed large increases from 1996 to 1998 (Table 3). Similar to trends observed for penicillin nonsusceptibility, sentinel groups had a high probability of detecting these increases in erythromycin nonsusceptibility (Table 3 ).

\section{Ability of Sentinel Groups To Detect Emerging Fluoroquinolone Resistance}

In 1998, seven isolates submitted to ABCs were nonsusceptible to levofloxacin; five of these were also nonsusceptible to trovafloxacin. The isolates came from seven different hospitals, located in five of the eight surveillance areas (California, Connecticut, Maryland, Minnesota, and Oregon). One of these hospitals, the only hospital from Oregon, had only five invasive pneumococcal isolates in 1998 and thus was excluded from our analysis of sentinel groups. Approximately $40 \%$ of sentinel groups of five laboratories in these areas (range 37\% in Connecticut to $45 \%$ in Maryland) included a laboratory with a fluoroquinolone-nonsusceptible isolate, except in California, where there was only one possible sentinel group of five laboratories and this group included the fluoroquinolone-nonsusceptible isolate.

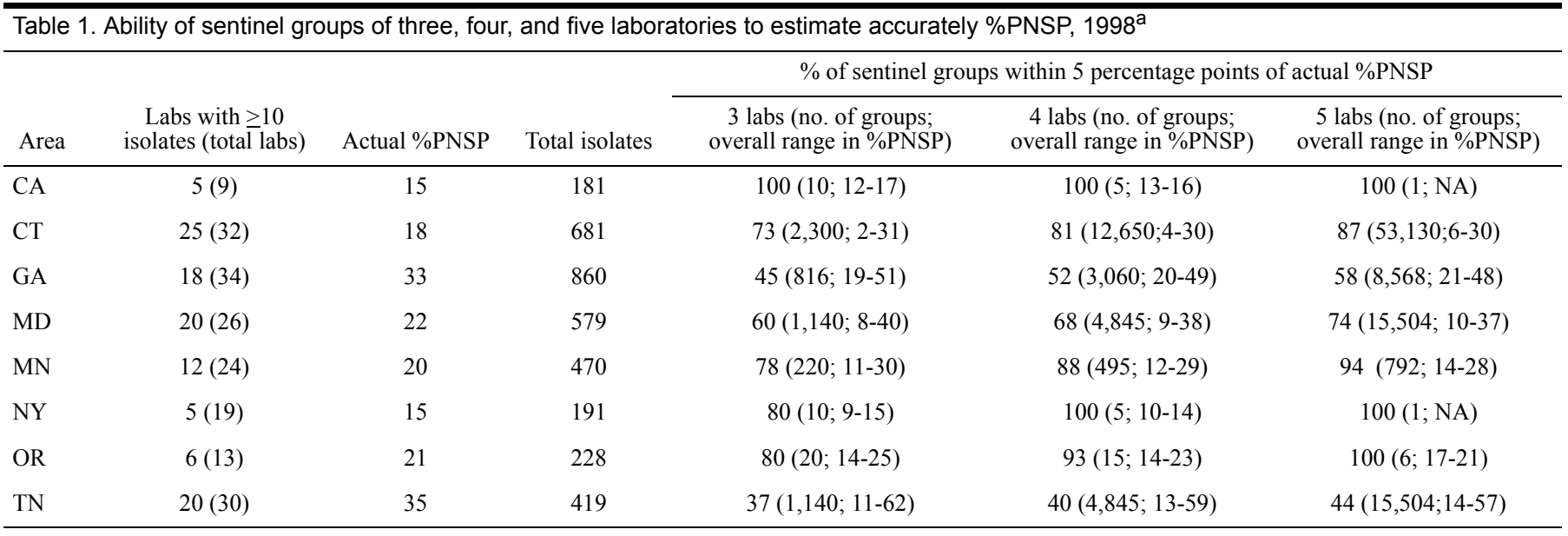

${ }^{\mathrm{a}}$ In Active Bacterial Core surveillance areas.

$\%$ PNSP, percent of penicillin-nonsusceptible invasive pneumococcal isolates. 

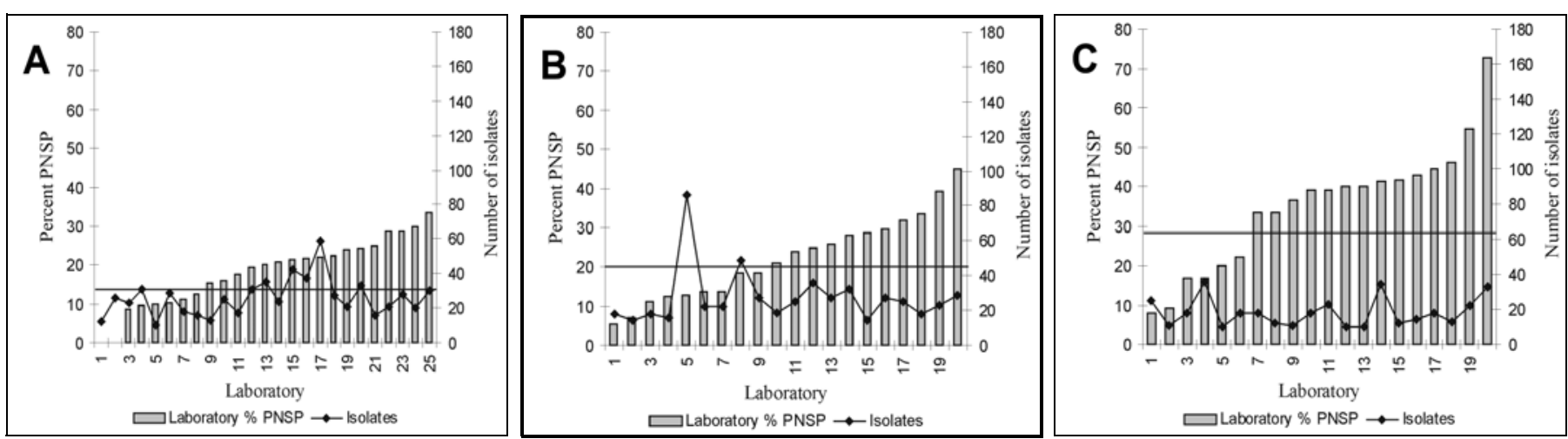

Figure. Between-laboratory variation in percent penicillin-nonsusceptible isolates (\%PNSP) and number of invasive pneumococcal isolates in selected surveillance areas. A) Connecticut; B) Selected counties of Maryland; C) Selected counties of Tennessee. Solid line denotes the area's actual \%PNSP from active, population-based surveillance.

\section{Evaluation of Hospital Predictors of \%PNSP}

The merged dataset of ABCs and AHA hospitals contained 104 hospitals: 24 (23\%) were in the high PNSP category, 52 (50\%) were in the average PNSP category; and 28 (27\%) were in the low PNSP category. Hospitals that admitted only children (four hospitals that matched between the two datasets) were significantly more likely to be in the high PNSP group than in the average group (all four hospitals fell in the high category; Fisher's exact test, $\mathrm{p}=0.008$ ). Larger hospitals (measured by adjusted inpatient days, total beds, or total beds set up and staffed) were more likely to fall in the average category, but this trend was not consistent for all indicators capturing hospital size (Table 4). Additional variables tested by univariate analysis were not predictive of falling in the high or low category (Table 4). When we performed similar analyses using the percent of erythromycin-nonsusceptible isolates or of isolates with resistance to more than one drug class as the primary outcome measure, no additional predictors were identified.

Table 2. Number of isolates required to estimate accurately \%PNSP in a given area and percentage of sentinel laboratory groups that met sample size requirements

\begin{tabular}{lccc}
\hline & $\begin{array}{c}\text { Actual } \\
\text { \%PNSP } \\
\text { Area } \\
\text { target range) }\end{array}$ & $\begin{array}{c}\text { No. of isolates } \\
\text { needed to esti- } \\
\text { mate \%PNSP }\end{array}$ & $\begin{array}{c}\% \text { of sentinel groups of 5 laborato- } \\
\text { ries with } \geq \text { no. of required isolates }\end{array}$ \\
\hline CA & $15(10-20)$ & 94 & 100 \\
CT & $18(13-23)$ & 172 & 3 \\
GA & $33(28-38)$ & 243 & 40 \\
MD & $22(17-27)$ & 183 & 12 \\
MN & $20(15-25)$ & 163 & 70 \\
NY & $15(10-20)$ & 97 & 100 \\
OR & $21(16-26)$ & 120 & 100 \\
TN & $35(30-40)$ & 191 & 0 \\
\hline
\end{tabular}

${ }^{a}$ No. of isolates, $n$, required to estimate the area's actual \%PNSP (P) within 5 percentage points $(d=0.05)$ with $95 \%$ confidence $(Z=1.96)$ is: $n=\left(Z^{2} P(1-P)\right) / d^{2}$, where $d$ is the range of accepted variation around the actual \%PNSP, and $\mathrm{Z}$ is the Z-score range within which values must fall. Because the total no. of isolates per area, N, was small, we corrected this estimate for finite population size: $n=n /[1+(n-1) / N]$. There is no power associated with this estimate (14).

$\%$ PNSP, percent of penicillin-nonsusceptible pneumocooccal isolates.
In areas where sentinel surveillance did not accurately estimate the \%PSNP (Georgia and Tennessee), can hospital predictors be used to improve performance? When we limited sentinel groups of five to the laboratories with the largest number of isolates, the range in \%PNSP narrowed, but accuracy was not guaranteed (range in Georgia 29\%-34\%; range in Tennessee $36 \%-44 \%$ ). Additionally, consistent with the analysis above, hand-picking sentinel hospitals to include those with a high proportion of pediatric isolates was likely to overestimate the actual \%PNSP; in Georgia the children's hospital had a \%PNSP of $61 \%$, whereas the area's true $\%$ PNSP was $33 \%$ (Table 1 ).

Table 3. Ability of sentinel groups of five laboratories to estimate an area's change in \%PNSP and erythromycin-nonsusceptible pneumococci, 1996-1998

\begin{tabular}{|c|c|c|c|c|}
\hline $\begin{array}{l}\text { Outcome } \\
\text { measure }\end{array}$ & Area $^{\mathrm{a}}$ & $\begin{array}{c}\text { Actual } \\
\text { change } \\
\text { in } \% \text { NS } \\
\text { pneumococci }\end{array}$ & $\begin{array}{l}\% \text { sentinel groups } \\
\text { within } 5 \text { percentage } \\
\text { points of the area's } \\
\text { actual change in } \% \\
\text { NS pneumococci }\end{array}$ & $\begin{array}{c}\% \text { of } \\
\text { sentinel groups } \\
\text { detecting an } \\
\text { increase or } \\
\text { decrease in the } \\
\text { actual \% NS } \\
\text { pneumococci }\end{array}$ \\
\hline \multirow[t]{6}{*}{ Penicillin NS } & CA & +3 & $100(1)$ & 100 \\
\hline & $\mathrm{CT}$ & +1 & $67(15,504)$ & \\
\hline & GA & -2 & $76(2,002)$ & \\
\hline & MD & +7 & $70(15,504)$ & 93 \\
\hline & $\mathrm{MN}$ & +6 & $97(252)$ & 99 \\
\hline & $\mathrm{TN}$ & 0 & $45(462)$ & \\
\hline \multirow{6}{*}{$\begin{array}{l}\text { Erythromycin } \\
\text { NS }\end{array}$} & CA & -2 & $100(1)$ & \\
\hline & $\mathrm{CT}$ & +2 & $95(15,504)$ & \\
\hline & GA & +6 & $80(2,002)$ & 86 \\
\hline & MD & +6 & $97(15,504)$ & 99 \\
\hline & $\mathrm{MN}$ & +7 & $83(252)$ & 99.6 \\
\hline & $\mathrm{TN}$ & +2.5 & $51(462)$ & -- \\
\hline
\end{tabular}

${ }^{\text {a }}$ NY joined ABCs in 1997; the only group of 5 laboratories in OR in 1996 did not match any of the groups in 1998 .

${ }^{\mathrm{b}}$ Groups that merged between the 2 years.

${ }^{\mathrm{c}}$ We limited this analysis to areas with $\geq 3 \%$ change in either direction.

$\%$ PNSP, percent penicillin-nonsusceptible pneumococci; NS, nonsusceptible. 
Table 4. Univariate analysis of characteristics of hospitals with a high or low \%PNSP compared with hospitals with an average \%PNSPa

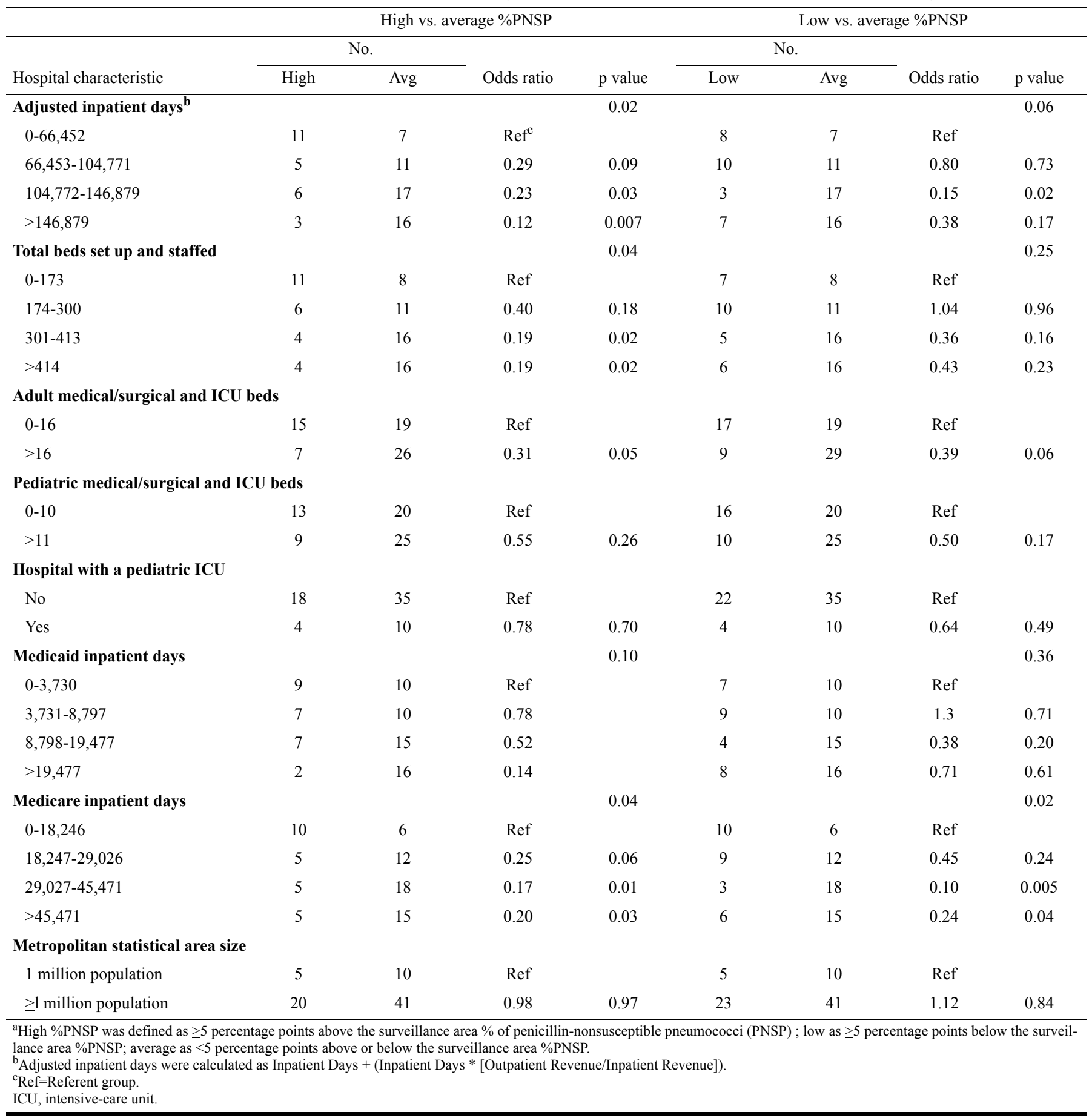

\section{Discussion}

As the incidence of drug-resistant pneumococcal disease continues to increase, the need for local and state-specific data on the emergence of drug-resistant invasive pneumococcal strains also grows. Although active, population-based surveillance provides highly accurate data for tracking pneumococcal resistance trends, few states can afford to implement such labor-intensive and costly systems. Moreover, states may have a variety of objectives for their surveillance systems, ranging from increasing awareness of resistance in local communities and promoting appropriate antibiotic use activities to estimating directly the drug-resistant isolates and trends in drug resistance; some of these objectives require more accurate surveillance systems than others.

Our evaluation of the performance of sentinel laboratory groups suggests that sentinel surveillance is a viable alternative to population-based surveillance in situations where a high degree of accuracy is not required. In some cases, sentinel 
surveillance may also be useful when accurate estimates of $\%$ PNSP trends are a primary objective. Sentinel laboratory groups were most reliable at detecting large increases or decreases in the proportion of nonsusceptible invasive isolates; the groups varied in their ability to predict an area's actual $\%$ PNSP; and they were poor at detecting newly emerging fluoroquinolone resistance. As a result, areas considering sentinel surveillance should design systems and interpret data with caution.

Baseline information on isolates processed annually per laboratory and between-laboratory variability in \%PNSP can be used to predict how well sentinel systems will perform at estimating this percentage in a given area. Such information can often be collected retrospectively or prospectively from microbiology laboratories. Authorities in areas with high between-laboratory variability or with few isolates per laboratory may want to consider alternatives or complements to sentinel systems.

Reasons for high between-laboratory variability in the proportion of nonsusceptible invasive pneumococcal isolates, such as we observed in Tennessee (Figure), remain unclear. This variability likely reflected differences in the risk for nonsusceptible pneumococcal infections in communities served by different laboratories. Because health insurance policies in the United States often determine the hospitals and laboratories that patients use, these facilities rarely serve populations that are representative of the community as a whole or even the neighborhood where the hospital is located. Characterizing risk factors for nonsusceptible invasive pneumococcal disease in a hospital's patient population is difficult. Readily obtainable hospital characteristics such as those collected by AHA did not explain the between-laboratory variation we observed. Unfortunately, some known predictors of resistance in healthcare settings, such as suburban middle- and upper-class patient populations $(15,16)$, were not available to link to our surveillance data.

Although most basic hospital characteristics were not a reliable guide to selecting laboratories to be included in sentinel systems, pediatric hospitals were significantly more likely than other hospitals in an area to have a high \%PNSP. Because children are a primary reservoir of S. pneumoniae and the incidence of invasive pneumococcal disease is elevated in children and the elderly (1), states may sometimes choose to include children's hospitals in sentinel surveillance systems to increase their likelihood of identifying resistance problems. However, to track trends in resistance to drugs such as fluoroquinolones that are not indicated for use in children, children's hospitals may not be reliable indicators.

For states wishing to increase the reliability of sentinel systems, increasing the overall number of laboratories participating in sentinel systems improved the accuracy of systems, particularly in areas where the \%PNSP approaches 50\%. However, in areas with high between-laboratory variation in \%PNSP, accuracy is difficult to achieve without including most laboratories in the system.
For states or regions with a primary objective of detecting rare, newly emerging resistance profiles, more than one surveillance approach may be necessary. For example, sentinel surveillance combined with universal reporting of fluoroquinolone- or vancomycin-resistant pneumococci will help detect important new strains before they become widespread. Additionally, authorities in such areas may consider collecting the isolates captured by sentinel facilities and conducting susceptibility testing by using a more diverse drug panel than is typically used in most clinical microbiology laboratories.

If used and interpreted appropriately, sentinel laboratory surveillance helps document pneumococcal resistance and improve prevention efforts. Evaluation of alternative surveillance methods such as analysis of hospital antibiograms (17) or direct electronic reporting of susceptibility results from hospital laboratories to a central network (M. Soriano-Gabarro, unpub. data) will further contribute to identifying low-cost, feasible methods of documenting trends in pneumococcal resistance.

\section{Acknowledgments}

We acknowledge B. Barnes, N. Barrett, W. Baughman, N. Bennett, J. Besser, P. Cieslak, A. Craig, P. Daily, B. Damaske, R. Facklam, M. Farley, L. Gelling, J. Hadler, L Harrison, T. Hilger, J. Jorgensen, L. Lefkowitz, C. Lexau, R. Lynfield, M. Pass, A Reingold, K. Robinson, G. Rothrock, K. Stefonek, C. Wright, and S. Zansky for collecting population-based surveillance for invasive pneumococcal disease. We are grateful to J.T. Weber and E. Brink for comments on the manuscript.

Dr. Schrag is an epidemiologist in the Respiratory Diseases Branch, Division of Bacterial and Mycotic Diseases at the Centers for Disease Control and Prevention. Her research focuses on methods of monitoring and preventing the spread of pneumococcal resistance and on prevention of neonatal sepsis.

\section{References}

1. Whitney C, Farley M, Hadler J, Harrison L, Lexau C, Reingold A, et al. Increasing prevalence of multidrug-resistant Streptococcus pneumoniae in the United States. N Engl J Med 2000;343:1917-24.

2. Klugman KP. Pneumococcal resistance to antibiotics. Clin Microbiol Rev 1990;3:171-96.

3. Heffelfinger JD, Dowell SF, Jorgensen JH, Klugman KP, Mabry LR, Musher DM, et al. Management of community-acquired pneumonia in the era of pneumococcal resistance: a report from the Drug-Resistant Streptococcus pneumoniae Therapeutic Working Group. Arch Intern Med 2000;160:1399-408.

4. Dowell SF, Butler JC, Giebink GS, Jacobs MR, Jernigan D, Musher DM, et al. Acute otitis media: management and surveillance in an era of pneumococcal resistance - a report from the Drug-Resistant Streptococcus pneumoniae Therapeutic Working Group. Pediatr Infect Dis J 1999;18:1-9.

5. Belongia E, Proctor M, Vandermause M, Ahrabi-Fard S, Knobloch M, Keller P, et al. Antibiotic susceptibility of invasive Streptococcus pneumoniae in Wisconsin, 1999. WMJ 2000;99:55-9.

6. Roush S, Birkhead G, Koo D, Cobb A, Fleming D. Mandatory reporting of diseases and conditions by health care professionals and laboratories. JAMA 1999;282:164-70.

7. Jernigan DB, Kargacin L, Poole A, Kobayashi J. Sentinel surveillance as an alternative approach for monitoring antibiotic-resistant invasive pneumococcal disease in Washington State. Am J Pub Health 2001;91:142-5. 
8. Richards MJ, Edwards JR, Culver DH, Gaynes RP. Nosocomial infections in medical intensive care units in the United States. Crit Care Med 1999;27:887-92.

9. Fleming DM, Zambon M, Bartelds AI, deJong JC. The duration and magnitude of influenza epidemics: a study of surveillance data from sentinel general practices in England, Wales and the Netherlands. Eur J Epidemiol 1999; $15: 467-73$

10. Massari V, Maions P, Desenclose JC, Flahault A. Six years of sentinel surveillance of hepatitis B in general practice in France. Eur J Epidemiol 1998;14:765-6.

11. Breiman RF, Butler JC, Tenover FC, Elliott JA, Facklam RR. Emergence of drug-resistant pneumococcal infections in the United States. JAMA 1994;271:1831-5.

12. Centers for Disease Control and Prevention. Geographic variation in penicillin resistance in Streptococcus pneumoniae-selected sites, United States, 1997. MMWR Morb Mortal Wkly Rep 1999;48:656-61.

13. National Committee for Clinical Laboratory Standards. Table 2G. MIC interpretive standards (micrograms $/ \mathrm{mL}$ ) for Streptococcus pneumoniae. Villanova (PA): The Committee; 2000.

14. Cochran WG. Sampling techniques. 2nd edition. New York: John Wiley and Sons; 1963.

15. Chen FM, Breiman RF, Farley M, Plikaytis B, Deaver K, Cetron MS. Geocoding and linking data from population-based surveillance and the US Census to evaluate the impact of median household income on the epidemiology of invasive Streptococcus pneumoniae infections. Am J Epidemiol 1998;148:1212-8.
16. Hofmann J, Cetron MS, Farley MM, Baughman WS, Facklam RR, Elliott JA, et al. The prevalence of drug-resistant Streptococcus pneumoniae in Atlanta. N Engl J Med 1995;333:481-6.

17. Chin AE, Hedberg K, Cieslak PR, Cassidy M, Stefonek KR, Fleming DW. Tracking drug-resistant Streptococcus pneumoniae in Oregon: an alternative surveillance method. Emerg Infect Dis 1999;5:688-93.

Address for correspondence: Stephanie Schrag, Respiratory Diseases Branch, MS C23, Division of Bacterial and Mycotic Diseases, Centers for Disease Control and Prevention, 1600 Clifton Rd., Atlanta, GA 30333, USA; fax: 404639-3970; e-mail: Zha6@cdc.gov

Research Studies: Articles should be 2,000 to 3,500 words and should include references, not to exceed 40. Use of subheadings in the main body of the text is recommended. Photographs and illustrations are encouraged. Provide a short abstract (150 words) and a brief biographical sketch of first author - both authors if only two.

These articles report laboratory and epidemiologic results within a public health perspective. Although these reports may be written in the style of traditional research articles, they should explain the value of the research in public health terms and place the findings in a larger perspective (e.g., "Here is what we found, and here is what the findings mean"). 